\title{
What defines mindfulness-based programs? The warp and the weft
}

\author{
R. S. Crane ${ }^{1 *}$, J. Brewer ${ }^{2,3}$, C. Feldman ${ }^{4}$, J. Kabat-Zinn ${ }^{2}$, S. Santorelli ${ }^{2}$, J. M. G. Williams ${ }^{5}$ and \\ W. Kuyken ${ }^{5}$ \\ ${ }^{1}$ Centre for Mindfulness Research and Practice, School of Psychology, Bangor University, Brigantia Building, Gwynedd, UK \\ ${ }^{2}$ Department of Medicine, Center for Mindfulness in Medicine, Health Care, and Society, University of Massachusetts Medical School, Worcester, \\ MA, USA \\ ${ }^{3}$ Yale University School of Medicine \\ ${ }^{4}$ Freelance Teacher and Writer, Totnes, Devon, UK \\ ${ }^{5}$ Oxford Mindfulness Centre, University Department of Psychiatry, University of Oxford, Warneford Hospital, Oxford, UK
}

There has been an explosion of interest in mindfulness-based programs (MBPs) such as Mindfulness-Based Stress Reduction (MBSR) and Mindfulness-Based Cognitive Therapy. This is demonstrated in increased research, implementation of MBPs in healthcare, educational, criminal justice and workplace settings, and in mainstream interest. For the sustainable development of the field there is a need to articulate a definition of what an MBP is and what it is not. This paper provides a framework to define the essential characteristics of the family of MBPs originating from the parent program MBSR, and the processes which inform adaptations of MBPs for different populations or contexts. The framework addresses the essential characteristics of the program and of teacher. MBPs: are informed by theories and practices that draw from a confluence of contemplative traditions, science, and the major disciplines of medicine, psychology and education; underpinned by a model of human experience which addresses the causes of human distress and the pathways to relieving it; develop a new relationship with experience characterized by present moment focus, decentering and an approach orientation; catalyze the development of qualities such as joy, compassion, wisdom, equanimity and greater attentional, emotional and behavioral self-regulation, and engage participants in a sustained intensive training in mindfulness meditation practice, in an experiential inquiry-based learning process and in exercises to develop understanding. The paper's aim is to support clarity, which will in turn support the systematic development of MBP research, and the integrity of the field during the process of implementation in the mainstream.

Received 7 June 2016; Revised 23 November 2016; Accepted 23 November 2016; First published online 29 December 2016

Key words: Fidelity, mindfulness-based cognitive therapy, mindfulness-based program, mindfulness-based stress reduction.

\section{Introduction}

There has been an explosion of interest in mindfulnessbased programs (MBPs) such as Mindfulness-Based Stress Reduction (MBSR) and Mindfulness-Based Cognitive Therapy (MBCT) in the last two decades. MBSR has accrued a robust evidence base in improving mental health outcomes in those with chronic physical health problems (Bohlmeijer et al. 2010). MBCT is an adaptation developed to teach those at high risk of depressive relapse skills to stay well and has been shown to be effective (Kuyken et al. 2016). There are multiple other MBPs with varying levels of research evidence at each stage of the research journey (Dimidjian \& Segal, 2015).

\footnotetext{
* Address for correspondence: R. S. Crane, Ph.D., M.A., Centre for Mindfulness Research and Practice, School of Psychology, Bangor University, Brigantia Building, Gwynedd LL57 2AS, UK.

(Email: r.crane@bangor.ac.uk)
}

For the sustainable development of the field there is a need to pause and address some fundamental questions. Dimidjian \& Segal (2015) analyzed the status of the extant evidence using the National Institutes of Health (NIH) Stage Model (Onken et al. 2014). This is a model of behavioral intervention development composed of six stages: basic science (Stage 0); intervention generation, refinement, modification, and adaptation and pilot testing (Stage I); traditional efficacy testing (Stage II); efficacy testing with real-world providers (Stage III); effectiveness research (Stage IV) and; dissemination and implementation research (Stage V). The mapping of the MBP evidence base onto this model by Dimidjian \& Segal clarifies that the evidence is heavily saturated in Stage I, lightly represented in Stages 0 and II, and that to date there is minimal research in Stages III, IV and V. Overall, in their analysis they identify significant strengths (e.g. breadth of clinical problems and populations targeted), and significant weaknesses (e.g. the need for an integrated 
and systematic approach to core research questions across all stages of the research from basic through to implementation science). In this editorial we focus on a critical underpinning issue throughout the Stage Model - namely the fidelity/integrity of the intervention itself. Fidelity is central to all stages but has particular salience at Stages I and V. Stage I involves the development of the intervention, which requires great clarity about for whom it is intended, and a theoretical exposition of what mechanisms are being targeted. It also requires careful consideration of what clinician training and supervision is required to deliver the intervention effectively. Stage V addresses implementation, which raises similar issues about what key aspects of fidelity need to be maintained when implementing in real world settings.

Given the proliferation of MBP development and research, there is a need to re-clarify the core ingredients of mindfulness-based programs, and the implications this has for professional training, supervision and implementation. This clarity is important for the field so that existing research can be meaningfully interpreted, future research uses agreed definitions and established protocols, MBP teachers are trained appropriately, and the general public are assured that programs titles accurately describe what is delivered.

\section{MBPs in context}

MBSR was developed as an education and training vehicle for people with chronic health problems and those suffering from the mounting demands associated with psychological and emotional stress, to learn to relate in new ways to life challenges (Kabat-Zinn, 2013). Other programs based upon the foundational approach and structure of MBSR have since been developed with particular aims across a broad range of settings, including hospitals, schools and prisons, and are gaining interest from policy makers (Ryan, 2013; Mindfulness All Party Parliamentary Group, 2015).

MBP developments are nested within a wider context of empirical developments within psychology, medicine, health care and education which include 'mindfulness-informed' programs such as Acceptance and Commitment Therapy (Hayes et al. 2011), Compassion Focused Therapy (Gilbert, 2009), Dialectical Behavioral Therapy (Linehan, 1993), Mindful Self Compassion (Neff \& Germer, 2013) and developments in the field of Positive Psychology (Seligman \& Csikszentmihalyi, 2000). These mindfulness-informed developments are part of the third wave of empirically tested psychotherapies (first wave being behavioral therapy; second wave being cognitive behavioral therapy). Third wave approaches have a decreased emphasis on controlling internal experience, and an increased emphasis on themes such as acceptance, metacognition and how people relate to their experiences. Mindfulness-informed programs share several underpinning theoretical ideas with MBPs, and many include some mindfulness meditation practice in their approach. However, a distinctive feature of MBPs is that systematic and sustained training in formal and informal mindfulness meditation practices (for both teacher and participants) is central both to the therapeutic approach and underpinning theoretical model. They are based on mindfulness.

It should also be noted that other MBPs exist alongside MBSR/MBCT which also share this emphasis on the practice of mindfulness-meditation as a central pedagogical component. An important example are 'second generation' mindfulness-based interventions which are openly spiritual in nature, make the linkage to the Buddhist teachings explicit within the pedagogy and are more traditional in the manner in which they construct and teach mindfulness (e.g. Van Gordon et al. 2015).These programs are at an earlier stage of development and research is now underway. However, the boundary to the analysis in this paper are what have retrospectively become termed 'first-generation' MBPs - i.e. MBSR and MBCT (which represent the strongest evidence within the field), and the range of programs which have developed out of these (e.g. Bowen et al. 2009; Duncan \& Bardacke, 2010; Kristeller et al. 2012). These first-generation MBPs, while drawing aspects of their underpinning models and practices from aspects of Buddhism, aim to clearly re-contextualize both the program content and theoretical underpinnings within the mainstream. That is to say, the aim is to ensure MBPs are based in science and contemporary approaches to managing mental and physical health and supporting well-being; that they are suitable for delivery in mainstream public institutions across a range of settings and cultures; and that they are maximally accessible to people with diverse values and religious affiliations.

It is also important to note that while MBPs have been developed for particular issues such as stress and depression, there are other empirically supported approaches for such difficulties. There is as yet no compelling evidence for specificity of hypothesized mechanisms or differential outcomes. This may be an artifact of the many shared components between these approaches, or it may simply be that this work has not yet been done. Our hope is that the clarity we aim to offer in this editorial will support measurement, mechanisms, and mediators research, as a recent editorial has argued is needed (Davidson, 2016).

\section{Development trajectory for MBPs}

In the context of high interest and rapid proliferation Dimidjian \& Segal (2015) identify several key 
challenges for the next phase of research developments. This editorial focuses on one of these challenges research and practice issues in the arena of MBP fidelity. If these challenges are not carefully addressed they will undermine the developing science as well as the quality and integrity of implementation in routine practice. Mindfulness training in various forms is rapidly being applied within different contexts and populations, and morphing into multiple curriculums of differing lengths and teaching methods. Mindfulness can be intuitively appealing to teachers and participants. There is consequently a grassroots spread of practice into populations and contexts, which at times is ahead of the evidence. The word mindfulness has become a word conveying a multitude of meanings and practices. This is a confusing context within which to systematically build the science and the practice integrity of MBPs.

\section{The essential and variable elements of first-generation MBPs}

We use the metaphor of 'warp and weft' to represent the 'fabric' of MBPs. In weaving, the warp is the term for the fixed thread that runs vertically through the cloth, while the weft is the term for the transverse thread that makes each tapestry unique. The warp is the thread that is fixed when the loom is set up, while the weft varies in texture and color. The essential, constant and integral threads that define an MBP regardless of population or context make up the 'warp'; they are what make it a mindfulness-based program. Each adapted MBP then introduces a unique 'weft' that seeks to target the training to a particular population and/or context. Within each aspect (program and teacher) we examine what is warp (i.e. essential to an MBP), and what is weft (i.e. will vary depending on context and/or population) (Table 1). The warp elements of the framework are anchored within the program forms of MBSR and MBCT. We suggest that the framework we offer can provide some unifying clarity for the wider MBP field on what an MBP is and is not.

\section{The essential ('warp') elements of MBPs}

\section{The MBP}

(1) Is informed by theories and practices that draw from a confluence of contemplative traditions, science, and the major disciplines of medicine, psychology and education

The key influences that have shaped MBPs are:

Contemplative mindfulness practices. MBPs are underpinned by theories about the human mind-body drawn from aspects of early Buddhist psychology, which articulate the ways in which people can come to recognize universal habitual psychological patterns that create and maintain distress. This philosophy and psychology is nuanced and complex. It includes a wide array of teachings and practices found across the breadth of both Buddhist and other traditions, which are beyond the scope of this paper (Williams \& Kabat-Zinn, 2013). However, there is high level consensus on common features found within the cultivation of mindfulness. These include that people can learn that habitual reactive patterns stem from unhelpful habits of the mind; that fear, denial and discrepancy-based thinking create and exacerbate distress; and that skillful ways of relating to experience can be developed through awareness, wise discernment and practice which offer the potential for (moments of) freedom from reactivity (Gethin, 1998).

MBPs address some universal features of human experience using mindfulness practices as a vehicle for a systematic training of the mind in the service of developing greater awareness of self and others, and thus, greater understanding. It is therefore not surprising that mindfulness practices can be found in many of the world's contemplative wisdom traditions. MBPs draw on aspects of these traditions while leaving behind their religious, esoteric and mystical elements. Crucially they are presented in an invitational way, asking people to use the mindfulness practices in whatever way best supports their aims and aspirations. The practices and frameworks employed within MBPs are thus re-contextualized for implementation within mainstream settings by ensuring that they are delivered in inclusive and culturally appropriate ways.

Science. The development of MBPs is grounded in developments in theory, cognitive neuroscience (Tang et al. 2015), translational research (Strauman \& Merrill, 2004; Meadows et al. 2014) and evidence-based practice across a range of disciplines. Empirical evidence informs the theoretical models underpinning MBP's aims and innovations, and continues to refine understanding of what works best, at what time, for which group of participants (Davidson, 2016).

Medicine. MBSR, the original MBP, was developed within a medical framework and culture, as a form of participatory medicine in which patients are challenged to discover and draw upon their own resources for learning, healing, and transformation (Kabat-Zinn, 2003).

Psychology. MBPs were introduced and incorporated into the field of psychology through the development of MBCT (Segal et al. 2013). Psychological theory and research now guides the development of many MBPs (Dimidjian \& Segal, 2015). Indeed, the field of cognitive 
Table 1. The essential (warp) and flexible (weft) ingredients of MBPS

Warp Weft

MBP

1. Is informed by theories and practices that draw from a confluence of contemplative traditions, science, and the major disciplines of medicine, psychology and education

2. Is underpinned by a model of human experience which addresses the causes of human distress and the pathways to relieving it

3. Develops a new relationship with experience characterized by present moment focus, decentering and an approach orientation

4. Supports the development of greater attentional, emotional and behavioral self-regulation, as well as positive qualities such as compassion, wisdom, equanimity.

5. Engages the participant in a sustained intensive training in mindfulness meditation practice, in an experiential inquiry-based learning process and in exercises to develop insight and understanding

MBP teacher

1. Has particular competencies which enable the effective delivery of the MBP

2. Has the capacity to embody the qualities and attitudes of mindfulness within the process of the teaching

3. Has engaged in appropriate training and commits to ongoing good practice

4. Is part of a participatory learning process with their students, clients or patients
1. The core essential curriculum elements are integrated with adapted curriculum elements, and tailored to specific contexts and populations

2. Variations in program structure, length and delivery are formatted to fit the population and context

1. Has knowledge, experience and professional training related to the specialist populations that the mindfulness-based course will be delivered to

2. Has knowledge of relevant underlying theoretical processes which underpin the teaching for particular contexts or populations science has to a large extent taken over the detailed work of investigating the precise mechanisms by which MBPs alleviate distress and enable flourishing. The process involves mapping theories and empirical understanding across a range of disciplines.

Education. The pedagogical processes employed in MBPs draw on educational principles that are experiential, interactive, participatory, student-centred and relationship-centred (Santorelli, 2000).

(2) Is underpinned by a model of human experience which addresses the causes of human distress and the pathways to relieving it

The confluence described in the previous section is supported by a model of distress that applies across MBPs that explains certain facets of how human distress is created and maintained, and how mindfulness training deals with these maintaining factors, and thus alleviates distress and supports mental health and well-being e.g. (Kabat-Zinn, 2013; Segal et al. 2013). It draws on aspects of cognitive science (e.g. attention and executive control and decentering Barnard \& Teasdale, 1991) and trans-diagnostic work (e.g. repetitive thought and experiential avoidance Dudley et al. 2011). This emerging model is being clarified and developed as empirical understanding is built (Brewer et al. 2013; Chiesa et al. 2013; Gu et al. 2015; Van der Velden et al. 2015). The field is young and emergent, and the underpinning frameworks are the subject of debate and empirical investigation.

(3) Develops a new relationship with experience characterized by present moment focus, decentering, and an approach orientation

An underpinning premise of the MBP model is the understanding that how people relate and respond to various forms of distress (rather than the distress itself) lies at the core of many problems and disorders. It is this that determines both the capacity and the extent to which distress can be relieved and transformed. The training aims to enable participants to recognize habitual, conditioned modes of reacting and make a radical shift in their relationship to their thoughts, 
feelings and body sensations, as well as to outer circumstances. The shift in relationship is based on what is called decentering (Segal et al. 2013) or re-perceiving (Shapiro et al. 2006), in which the MBP participant is trained to attend to thoughts and feelings as mental events by noticing how they come and go in the mind and how each has consequences in the very next moment. For example, a person who is rushing to a meeting, might come to realize that in addition to the stress of being late they are compounding the stress by the thought 'if I'm late everybody will think I am not coping'. Whereas in an approach such as cognitive therapy, a person might be taught to challenge such a thought, in MBPs the emphasis is on seeing it as a mental event, noticing its effects on the body, and the way it creates further feelings and thoughts that were not part of the original situation. Gradually people come to see that their thoughts are not necessarily valid representations of reality.

(4) Supports the development of greater attentional, emotional and behavioral self-regulation, as well as positive qualities such as compassion, wisdom, equanimity

Attentional training within MBPs offers a foundation for the attitudinal dimensions of mindfulness to emerge. MBPs seek to support the ability to meet experience with curiosity, equanimity and compassion (Feldman \& Kuyken, 2011). An experiential understanding develops that pain is an inherent part of human experience and is ever changing. An orientation of mind characterized by curiosity, patience and equanimity supports the development of compassion and wisdom. There is an intention to cultivate an internal climate of friendliness towards experience whether it be pleasant or unpleasant. This supports a shift away from habitually battling with experience. MBPs also aim to cultivate positive qualities such as joy, and the ability to recognize and savour nourishing and pleasant experiences.

(5) Engages the participant in a sustained intensive training in mindfulness meditation practice, in an experiential inquiry-based learning process, and in exercises to develop insight and understanding

Direct experiential, systematic and intensive engagement in formal and informal mindfulness meditation practices is foundational to teaching/learning in MBPs. The training develops familiarity with and understanding of the mind and body, and appreciation that attention can be regulated, fine-tuned, and optimized through training. Rather like physical training regimes, the training progresses developmentally and sequentially throughout the program.

The integrity and fidelity of each MBP is maintained by ensuring that all the curriculum elements for the particular program are included as intended (adherence) and that curriculum elements are not added which do not belong to the particular program form (differentiation) (Crane et al. 2013). It is critical that the curriculum employed and the particular MBP title match each other. If there is divergence from an established curriculum, teaching structure or process then a new title for the adaptation should be employed, and research should clearly identify the adapted curriculum guide used, and if any checks on fidelity and adherence were employed.

Adherence to program form is supported by protocols for each MBP (e.g. see Santorelli et al. 2017 and Stahl \& Goldstein, 2010, for MBSR curriculum guides, and Segal et al. 2013, for the MBCT curriculum guide). Other MBPs have published guides which specify their particular curriculum adaptations. MBP curriculums vary in terms of number and duration of sessions and time commitments for home practice. For example, MBSR is scheduled over eight weekly sessions lasting $2.5 \mathrm{~h}$ with $40 \mathrm{~min}$ of guided mindfulness meditation home practice per day. MBCT is the same but with $2 \mathrm{~h}$ long sessions. There is not scope to fully list the curriculum elements and therapeutic techniques which, if included, would be regarded as outside the model for a particular first generation MBP (the differentiation aspect of program integrity). However, examples include: thought challenging and collecting evidence for and against the truth for thoughts (which is part of cognitive therapy); an orientation of problem solving or fixing; different sorts of meditation practices (e.g. mantra meditation); talking about the detailed history of participants; and an emphasis on relaxation training.

MBPs typically include mindfulness training via three formal mindfulness meditation practices - the body scan, mindful movement and sitting meditation. Practices are assigned as daily home practice with the support of recorded guidance. Throughout the program participants are also encouraged to generalize through informal practice by bringing awareness in particular ways to everyday activities.

MBPs include learning experiences built around the formal mindfulness meditation training. These include engaging in an individual and collective investigative inquiry to develop skills in recognizing direct experiences (i.e. thoughts, emotions and sensations), patterns of relating to experience, and the wider implications of the understandings that emerge. This externalized teacher- led interactional process in class becomes a representation of the work that the MBP participants begin to engage in within themselves, whereby the investigative process becomes 'internalized'. Foci for inquiry include participants' direct experience of meditation practices, and exploration of pleasant and 
unpleasant experiences which has implications for recognizing established patterns of reactivity and the possibility of responding differently. The focus will depend on the context/population, but MBPs particularly investigate the territory of difficult, unwanted experiences - stress, depression, physical pain, etc. Participants are invited to explore 'How am I experiencing and relating to these difficulties physically, emotionally and psychologically? What possibilities are there for stepping out of habitual aversive reactive patterns into responding with greater awareness and agency?' Each MBP employs particular exercises to facilitate these investigations.

\section{The teacher}

(1) Has particular competencies which enable the effective delivery of the $M B P$

MBP teachers have particular 'explicit' or 'visible' teaching competencies. These competencies are described and can be assessed using the Mindfulness-Based Interventions: Teaching Assessment Criteria (MBI:TAC), which has shown promising evidence as a reliable and valid tool (Crane et al. 2012, 2013, 2016). The six teaching competence domains within the MBI:TAC are coverage, pacing and organization of the session curriculum; specific interpersonal relational skills; skilful guiding of formal mindfulness meditation practices; specific methodologies for conveying the course themes through interactive inquiry, group dialog and didactic teaching; and effective holding of the group teaching/learning environment. These require particular tailored training experiences to develop (Crane et al. 2010).

\section{(2) Has the capacity to embody the qualities and attitudes of mindfulness within the process of the teaching}

MBP teachers have in common a sustained commitment to cultivating mindfulness through regular daily formal and informal mindfulness practices in everyday life. The way in which mindfulness practice manifests in the life of the teacher and is tangibly sensed in the MBP classroom is termed 'embodiment'. The theoretical model hypothesizing how MBP training has its effects includes embodiment as a critical factor in enabling participants to move towards experiential (rather than conceptual) knowing of mindfulness (Teasdale et al. 2002). Embodiment is a natural outcome of the teacher's intention to mindfully inhabit their experience in everyday life and in the MBP classroom, to whatever degree possible in any given moment. It is neither a striving for a particular state, nor a contrivance to artificially fabricate a particular persona, mask or appearance. Through their own personal mindfulness practice, teachers naturally come to inhabit the qualities associated with mindfulness (i.e. non-striving, non-judging, allowing), and the understandings and ethics underpinning the practices. The embodied practice element of ethics within MBPs are thus emergent and cultivated through the practice rather than being mandated. The practice of mindfulness enables the participant to try out the possibility of eliciting kindness, openness, and patience toward immediate experience, whether the experience is perceived as pleasant, unpleasant, or neutral (Grossman, 2015). MBP teacher training aims to cultivate the capacity in teachers to communicate mindfulness through the process as much as through the content of the teaching, by integrating training in formal and informal mindfulness practices into the other aspects of the teacher education and training curriculum.

\section{(3) Has engaged in appropriate training and commits to ongoing good practice}

MBP teachers have by necessity engaged in appropriate education and training to build and deepen their teaching competence (Crane et al. 2010, 2012; Kabat-Zinn et al. 2011; Marx et al. 2015). Training involves foundational experiential engagement with mindfulness practice and with the particular MBP curriculum the teacher training is embedded within, preliminary teacher training to build core skills, knowledge and attitudes, and then further training to enable the practitioner to reach the threshold of competence. Within some training programmes, the training journey culminates in an assessment of teaching competence using the MBI:TAC (Crane et al. 2016), conducted by trainers who have been trained to reliably use the tool.

Teachers then commit to ongoing good professional practice in line with consensually agreed good practice standards and guidelines (UK Network for Mindfulness-Based Teacher Training Organisations, 2012; Santorelli, 2014). Two key elements of good practice are supervision (Evans et al. 2014), and ongoing engagement with a personal mindfulness practice including periodic periods of intensive mindfulness practice in a residential setting. As the work of first generation MBPs is becoming more established in the mainstream, more opportunities are opening up for residential mindfulness intensives within a thoroughly mainstream context (e.g. Mindfulness Network CIC, 2016).

In the UK there is a national listing of MBP teachers who have undertaken training to minimum good practice level, and are adhering to ongoing good practice (UK Network for Mindfulness-Based Teacher Training Organisations, 2016). In the USA there is an MBSR Registry for certified teachers (Centre for Mindfulness in Medicine Health Care and Society, 2016). The organization of governance within this emerging field to 
support the general public to access well prepared and qualified MBP teachers is underway.

MBP teachers operate within professional ethical codes anchored within their root profession (i.e. medicine, clinical psychology, teaching, etc.) (Baer, 2015), and appropriate to the ethos and ethics of the mainstream public institution within which they are implementing (Crane, 2016).

(4) Is part of a participatory learning process with their students, clients or patients

Delivery of MBPs is usually in a group context which supports perspective taking and the transition from personal story into investigation of common pathways which lead to distress. MBP teachers embed themselves within this investigation. They are in a particular form of relationship with their participants which is underpinned by recognition of our 'common humanity' (shared human experience) (Neff, 2011), and thus the mutual nature of the learning process (Santorelli, 2000).

\section{Integration of tailored elements into adapted mindfulness-meditation based programs}

As the field has developed, new MBPs have woven in a weft that aims to make the mindfulness framework and practices more accessible and useful for particular populations and in varying contexts. However it remains an empirical question for each new adaptation whether it improves the accessibility and usefulness of MBPs, and each adaptation needs to commit to building its own evidence base.

Adaptations are typically of three kinds:

(1) Adaptations informed by theoretical frameworks or models that are particular to a new population or setting. For example, MBCT for recurrent depression is oriented around a cognitive model of depressive relapse and how mindfulness can free the mind from engrained patterns of depressive rumination (Teasdale \& Chaskalson, 2011).

(2) Adaptations to the program that make it more accessible, acceptable and potent for a population. For example, Mindfulness-Based Eating Awareness Training employs mindful eating practices (Kristeller \& Wolever, 2011); mindful parenting programs use parenting activities and examples (Bogels et al. 2010).

(3) Adaptations to the program that embed MBPs in a particular context or setting. For example, MBSR is an outpatient program that can be offered in hospital outpatient settings; mindfulness in schools programs are adapted for delivery within school curricula (Kuyken et al. 2013); and mindfulness in organizations are adapted so as be accessible to and within businesses (Chaskalson, 2011).

We next identify the program and teacher characteristics that characterize the weft of MBPs, namely the ways they are adapted (see Table 1).

\section{Adapted MBP considerations}

(1) The core essential curriculum elements are integrated with adapted curriculum elements, and tailored to specific contexts and populations

It is important that there is explicit clarity regarding the core intentions of the MBP for the specific context and targeted participants. MBP developers should base the development in a clearly articulated aim and intention regarding the benefit and relevance of the program for a particular context and/or population (Teasdale et al. 2003; Dobkin et al. 2013). The adaptation needs to be grounded in a theoretically informed analysis of the specific mechanisms influencing distress or life themes in the target population and an analysis of the contextual issues involved in offering an MBP in a particular setting (e.g. schools, business, the military). This analysis is based on an understanding that general vulnerability (characteristics of the human condition that predispose us to distress), and specific vulnerabilities (particular patterns, traits or tendencies) co-exist (Williams, 2008). Adapted MBPs are tailored towards recognition of a specific vulnerability or life circumstance that characterizes a particular population (e.g. the easily triggered negative thinking of people vulnerable to recurrent depression). How do these issues present themselves? What are their characteristics? How are they triggered and maintained? The MBP developers then need to develop and seamlessly integrate the adapted 'weft' elements into the program to ensure that the curriculum skillfully maps onto these processes. They need to pose the question: 'What are the particular teaching processes and specific curriculum elements that may be required to support the mindfulness-based learning experience in this specific population/context?' Program development should include recognition of and a skillful response to any tensions or challenges that need to be considered in a particular context/population (for example, participants who may become suicidal Williams et al. 2015), so that there is an assurance that there is clearly an added value of the adaptation over existing MBPs. Dimidjian \& Segal (2015) caution that the rapid proliferation of new potential contexts and populations risks neglecting the importance of foundational specification of clear intervention targets and mediating processes of change on which subsequent research can build. 
(2) Variations in program structure, length and delivery are formatted to fit the population and context

The program form may also be changed to enable accessibility and tailoring for particular populations (e.g. shorter, but more sessions for chronic fatigue; half-day sessions, at fortnightly intervals to enable working people traveling from a distance to attend; individual delivery to those who cannot attend a group), and contexts (e.g. short sessions at lunch time for workplace mindfulness courses). There is increasing interest in digital delivery methods such as smartphone apps (Brewer et al. 2013), and some early evidence that these and other forms of lower intensity and self-help programs may be beneficial. However, more research is needed (Cavanagh et al. 2014).

Critically, it is important that new adaptations making use of new formats are given appropriate titles to clearly distinguish them from established programs. Such careful description and delineation is key to maintain the health and vitality of the emerging MBP field. Without it, the scientific literature will inevitably conflate studies of programs that may not be characteristic of the core structure, form, dose and delivery method of a particular MBP but represent themselves as such, and thus confound our ability to interpret the emerging scientific exploration of MBPs. It is also key to ensuring the public are given clear information on what program they are attending, what training their MBP teacher has had, and what they are (and are not) trained to teach.

\section{Teacher considerations for adapted programs}

In addition to all the 'warp' elements of training and ongoing adherence to good practice, teachers who are teaching particular curriculums, populations, or in particular contexts need congruent particular trainings. For example, if the teacher is delivering an MBP in a specific clinical setting, particular clinical training and experience in that specific domain is required. Teachers also need explicit training in the theoretical principles that underpin the adaptation so that they can effectively convey this essential element in their teaching.

\section{Conclusions}

This editorial sets out a framework to define the essential (warp) and variant (weft) ingredients of MBPs. Clarity and precision are needed both to maintain the integrity of the original programs and to support ongoing research into new programs, innovations, and developments. The current dialog between modern science and ancient contemplative wisdom traditions represents an extraordinary confluence of two epistemologies, interacting, learning from and informing one another. This confluence is proving extraordinarily creative - there is an upsurge of mindfulness-informed and mindfulness-based programs and approaches across medicine, psychology, and psychotherapy, and beyond, into business and education. There are also inherent tensions to navigate in the process of integrating paradigmatically different disciplines (Harrington \& Dunne, 2015; Crane, 2016).

The MBP field is at an early stage of development. The evidence supporting their efficacy in the treatment of physical and psychological health challenges in some areas is strong and building (e.g. see recent meta-analyses and reviews Carlson, 2012; Khoury et al. 2013; Kuyken et al. 2016). However, in many areas, the landscape can be best characterized as promising or as yet unexplored. For the field to realize its potential to maximally impact individual and societal health and well-being, there is a continued need for high quality innovation and research to investigate mechanisms, effectiveness, and implementation. Clarity regarding fidelity to program form and substance is an essential underpinning to ongoing research and practice development within this nascent and highly promising field.

\section{Acknowledgements}

The paper was written without external funding. We are grateful to Trish Bartley, Cindy Cooper, Susannah Crump and Petra Meibert who provided comments on the manuscript.

\section{Declaration of Interest}

R.C., J.K.Z., C.F., S.S., and J.M.G.W. receive royalties from mindfulness books they have authored. W.K. is Director of the Oxford Mindfulness Centre and Principal Investigator of several NIHR and Wellcome Trust research projects. J.M.G.W., C.F. and J.K.Z. receive freelance payments for training workshops and presentations related to mindfulness. R.C. is Director of the Centre for Mindfulness Research and Practice in Bangor, and a non-salaried Director of a not for profit company providing services for mindfulness-based teachers. S.S. is Executive Director and Director of the MBSR Clinic, and J.B. the Director of Research at the Center for Mindfulness in Medicine, Health Care, and Society, University of Massachusetts Medical School. J.K.Z. and J.M.G.W. were previous directors of the Center for Mindfulness in Medicine, Health Care, and Society, University of Massachusetts Medical School and the Oxford Mindfulness Centre respectively. J.K.Z. receives royalties from mindfulness-based books and guided meditation programs he has authored, and is the owner of a web-based outlet for sale of the guided 
meditations. S.F.S. is the Sole Proprietor of Guest-House Tapes and CDs.

\section{References}

Baer RA (2015). Ethics, values, virtues, and character strengths in mindfulness-based interventions: a psychological science perspective. Mindfulness 6, 956-969.

Barnard P, Teasdale JD (1991). Interacting cognitive subsystems: a systemic approach to cognitive-affective interaction and change. Cognition and Emotion 5, 1-39.

Bogels SM, Lehtonen A, Restifo K (2010). Mindful parenting in mental health care. Mindfulness 1, 107-120.

Bohlmeijer E, Prenger R, Taal E, Cuijpers P (2010). Meta-analysis on the effectiveness of mindfulness-based stress reduction therapy on mental health of adults with a chronic disease: what should the reader not make of it? Journal of Psychosomatic Research 69, 614-615.

Bowen S, Chawla N, Collins SE, Witkiewitz K, Hsu S, Grow J, Clifasefi S, Garner M, Douglass A, Larimer ME, Marlatt A (2009). Mindfulness-based relapse prevention for substance use disorders: a pilot efficacy trial. Substance Abuse 30, 295-305.

Brewer JA, Elwafi HM, Davis JH (2013). Craving to Quit: psychological models and neurobiological mechanisms of mindfulness training as treatment for addictions. Psychology of Addictive Behaviors 27, 366-379.

Carlson L (2012). Mindfulness-Based Interventions for physical conditions: a narrative review evaluating levels of evidence. International Scholarly Research Network 2012, 651583.

Cavanagh K, Strauss C, Forder L, Jones F (2014). Can mindfulness and acceptance be learnt by self-help?: a systematic review and meta-analysis of mindfulness and acceptance-based self-help interventions. Clinical Psychology Review 34, 118-129.

Centre for Mindfulness in Medicine Health Care and Society (2016) 2016, last update, Find an MBSR program near you (http://www.umassmed.edu/cfm/stress-reduction/ find-an-mbsr-program/).

Chaskalson M (2011). The Mindful Workplace: Developing Resilient Individuals and Resonant Organizations with MBSR, 1st edn. Wiley-Blackwell: UK.

Chiesa A, Serretti A, Jakobsen JC (2013). Mindfulness: topdown or bottom-up emotion regulation strategy? Clinical Psychology Review 33, 82-96.

Crane RS (2016). Implementing Mindfulness in the Mainstream: making the path by walking it. Mindfulness.

Crane RS, Eames C, Kuyken W, Hastings RP, Williams JMG, Bartley T, Evans A, Silverton S, Soulsby JG, Surawy C (2013). Development and validation of the Mindfulness-Based Interventions - Teaching Assessment Criteria (MBI:TAC). Assessment 20, 681-688.

Crane RS, Kuyken W, Hastings R, Rothwell N, Williams JMG (2010). Training teachers to deliver mindfulness-based interventions: learning from the UK experience. Mindfulness 1, 74-86.

Crane RS, Kuyken W, Williams JMG, Hastings R, Cooper L, Fennell MJV (2012). Competence in teaching mindfulness-based courses: concepts, development, and assessment. Mindfulness 3, 76-84.

Crane RS, Soulsby JG, Kuyken W, Williams JMG, Eames C (2016) 2016-last update, The Bangor, Exeter \& Oxford Mindfulness-Based Interventions Teaching Assessment Criteria (MBI-TAC) for assessing the competence and adherence of mindfulness-based class-based teaching (https://www.bangor.ac.uk/mindfulness/documents/ MBI-TACmanualsummaryaddendums05-16.pdf).

Davidson RJ (2016). Mindfulness-based cognitive therapy and the prevention of depressive relapse measures, mechanisms, and mediators. JAMA Psychiatry 73, 547-548.

Dimidjian S, Segal ZV (2015). Prospects for a clinical science of mindfulness-based intervention. American Psychologist 70, 593-620.

Dobkin PL, Hickman S, Monshat K (2013). Holding the heart of mindfulness-based stress reduction: balancing fidelity and imagination when adapting MBSR. Mindfulness 5, 710-718.

Dudley R, Kuyken W, Padesky CA (2011). Disorder specific and trans-diagnostic case conceptualisation. Clinical Psychology Review 31, 213-224.

Duncan LG, Bardacke N (2010). Mindfulness-based childbirth and parenting education: promoting family mindfulness during the perinatal period. Journal of Child and Family Studies 19, 190-202.

Evans A, Crane RS, Cooper L, Mardula J, Wilks J, Surawy C, Kenny M, Kuyken W (2014). A framework for supervision for mindfulness-based teachers:a space for embodied mutual inquiry. Mindfulness 6, 572-581.

Feldman C, Kuyken W (2011). Compassion in the landscape of suffering. Contemporary Buddhism 12, 143-155.

Gethin R (1998). The Foundations of Buddhism. Oxford University Press: USA.

Gilbert P (2009). The Compassionate Mind. Constable: London.

Grossman P (2015). Mindfulness: awareness informed by an embodied ethic. Mindfulness 6, 17-22.

Gu J, Strauss C, Bonda R, Cavanagh K (2015). How do mindfulness-based cognitive therapy and mindfulnessbased stress reduction improve mental health and wellbeing? A systematic review and meta-analysis of mediation studies. Clinical Psychology Review 37, $1-12$.

Harrington A, Dunne JD (2015). When mindfulness is therapy: ethical qualms, historical perspectives. American Psychologist 70, 621-631.

Hayes SC, Strosahl KD, Wilson KG (2011). Acceptance and Commitment Therapy: The Process and Practice of Mindful Change. Guilford Press: New York.

Kabat-Zinn J (2003). Mindfulness-based interventions in context: past, present, and future. Clinical Psychology: Science and Practice 10, 144-156.

Kabat-Zinn J (2013). Full Catastrophe Living: Using the Wisdom of Your Body and Mind to Face Stress, Pain and Illness. Delacorte: New York.

Kabat-Zinn J, Santorelli S, Blacker M, Brantley J, Meleo-Meyer F, Grossman P, Kesper-Grossman U, Reibel D, Stahl R (2011) 2011-last update, Training Teachers to Deliver Mindfulness-Based Stress Reduction Principles and Standards. (http://www.umassmed.edu/cfm/ trainingteachers/index.aspx). [18 March, 2011]. 
Khoury B, Lecomte T, Fortin G, Masse M, Therien P, Bouchard V, Chapleau M, Paquin K, Hofmann S, G. (2013). Mindfulness-based therapy: a comprehensive meta-analysis. Clinical Psychology Review 33, 763-771.

Kristeller JL, Wolever RQ (2011). Mindfulness-based eating awareness training for treating binge eating disorder: the conceptual foundation. Journal of Eating Disorders 19, 49-61.

Kuyken W, Warren FC, Taylor RS, Whalley B, Crane C, Bondolfi G, Hayes R, Huijbers M, Ma H, Schweizer S, Segal Z, Speckens A, Teasdale JD, Van Heeringen $K$, Williams M, Byford S, Byng R, Dalgleish T (2016). Efficacy and moderators of mindfulness-based cognitive therapy (MBCT) in prevention of depressive relapse: an individual patient data meta-analysis from randomized trials. Journal of the American Medical Association: Psychiatry 73, 565-574.

Kuyken W, Warren F, Taylor RS, Whalley B, Crane C, Bondolfi G, Dalgleish T (2016). Efficacy and moderators of mindfulness-based cognitive therapy (MBCT) in prevention of depressive relapse: an individual patient data meta-analysis from randomized trials. Journal of the American Medical Association: Psychiatry.

Kuyken W, Weare K, Ukoumunne OC, Vicary R, Motton N, Burnett R, Cullen C, Hennelly S, Huppert F (2013). Effectiveness of the Mindfulness in Schools Programme: non-randomised controlled feasibility study. British Journal of Psychiatry 203, 126-131.

Linehan M (1993). Skills Training Manual for Treating Borderline Personality Disorder: Diagnosis and Treatment of Mental Disorders. Guilford Press: New York.

Marx R, Strauss C, Williamson C (2015). Mindfulness apprenticeship: a new model of NHS-based MBCT teacher training. Mindfulness 6, 253-263.

Meadows GN, Shawyer F, Enticott JC, Graham AL, Judd F, Martin PR, Piterman L, Segal ZV (2014).

Mindfulness-based cognitive therapy for recurrent depression: a translational research study with 2-year follow-up. Australian and New Zealand Journal of Psychiatry 48, 743-755.

Mindfulness all Party Parliamentary Group (2015) 2015, last update, Mindful Nation UK (http://www.themind fulnessinitiative.org.uk/images/reports/MindfulnessAPPG-Report_Mindful-Nation-UK_Oct2015.pdf).

Mindfulness Network CIC (2016) 2016-last update, Retreats for mindfulness-based teachers (https://www. mindfulness-network.org/retreats.php).

Neff K (2011). Self-Compassion: Stop beating yourself and leave insecurity behind. HarperCollins: New York.

Neff K, Germer CK (2013). A pilot study and randomized controlled trial of the mindful self-compassion program. Journal of Clinical Psychology 1, 28-44.

Onken LS, Carroll KM, Shoham V, Cuthbert BN, Riddle M (2014). Re-envisioning clinical science: unifying the discipline to improve the public health. Clinical Psychological Science 2, 22-34.

Ryan T (2013). A Mindful Nation: How a Simple Practice Can Help Us Reduce Stress, Improve Performance, and Recapture the American Spirit. Hay House: USA.

Santorelli S (2000). Heal Thy Self. Crown Publications: USA.
Santorelli S (2014) 2014, last update, mindfulness-based stress reduction (MBSR): standards of practice (https://www. umassmed.edu/contentassets/24cd221488584125835e 2eddce7dbb89/mbsr_standards_of_practice_2014.pdf).

Santorelli SF, Kabat-Zinn J, Blacker M, Meleo-Meyer F, Koerbel L (eds) (2017) Mindfulness-Based Stress Reduction (MBSR) Authorized Curriculum Guide (http://www. umassmed.edu/cfm/training/mbsr-curriculum).

Segal ZV, Williams JMG, Teasdale JD (2013). Mindfulness-Based Cognitive Therapy for Depression. Guilford: New York.

Seligman M, Csikszentmihalyi M (2000). Positive psychology: an introduction. American Psychologist 55, 5-14.

Shapiro SL, Carlson LE, Astin JA, Freedman B (2006). Mechanisms of mindfulness. Journal of Clinical Psychology 62, 373-386.

Stahl B, Goldstein E (2010). A Mindfulness-Based Stress Reduction Workbook. New Harbinger: Oakland, CA.

Strauman TJ, Merrill TA (2004). The basic science/clinical science interface and treatment development. Clinical Psychology Science and Practice 11, 263-266.

Tang YY, Holzel BK, Posner MI (2015). The neuroscience of mindfulness meditation. Nature Reviews Neuroscience 16, 213-225.

Teasdale JD, Chaskalson M (2011). How does mindfulness transform suffering? II: the transformation of dukkha. Contemporary Buddhism 12, 103-124.

Teasdale JD, Moore RG, Hayhurst H, Pope M, Williams S, Segal ZV (2002). Metacognitive awareness and prevention of relapse in depression: empirical evidence. Journal of Consulting and Clinical Psychology 70, 275-287.

Teasdale JD, Segal ZV, Williams JMG (2003). Mindfulness training and problem formulation. Clinical Psychology: Science and Practice 10, 157-160.

UK Network for Mindfulness-Based Teacher Training Organisations (2012) 2012, last update, Good Practice Guidance for teaching mindfulness-based courses (http:// mindfulnessteachersuk.org.uk/).

UK Network for Mindfulness-Based Teacher Training Organisations (2016) 2016-last update, Listing of mindfulness-based course teachers (https://www. mindfulness-network.org/listingspagenew.php).

Van Der Velden AM, Kuyken W, Wattar U, Crane C, Pallesen KJ, Dahlgaard J, Fjorback LO, Piet J (2015). A systematic review of mechanisms of change in mindfulness-based cognitive therapy in the treatment of recurrent major depressive disorder. Clinical Psychology Review 37, 26-39.

Van Gordon W, Shonin E, Griffiths MD (2015). Towards a second generation of mindfulness-based interventions. Australian and New Zealand Journal of Psychiatry 49, 591-592.

Williams JMG (2008). Mindfulness, depression and modes of mind. Cognitive Therapy Research 32, 721-733.

Williams JMG, Fennell MJV, Barnhofer T, Crane RS, Silverton S (2015). Mindfulness and the Transformation of Despair: Working with People at Risk of Suicide. Guilford: USA.

Williams JMG, Kabat-Zinn J (eds) (2013). Mindfulness: Diverse Perspectives on its Meaning, Origins and Applications. Routledge. 